\title{
Diet Drink Consumption and the Risk of Cardiovascular Events: A Report from the Women's Health Initiative
}

\author{
Ankur Vyas, $M D^{7}$, Linda Rubenstein, $P h D^{2}$, Jennifer Robinson, $M D, M P H^{1,2}$, \\ Rebecca A. Seguin, PhD, CSCS 3 , Mara Z. Vitolins, DrPH, MPH, RD', \\ Rasa Kazlauskaite, MD, MSC, FACE $E^{5,6}$, James M. Shikany, DrPH ${ }^{7}$, Karen C. Johnson, MD, MPH', \\ Linda Snetselaar, RD, $P h D^{2}$, and Robert Wallace, MD, MSc 2,9

\begin{abstract}
'Division of Cardiovascular Medicine, University of lowa Hospitals \& Clinics, lowa City, IA, USA; ${ }^{2}$ Department of Epidemiology, College of Public Health, University of lowa, lowa City, IA, USA; ${ }^{3}$ Division of Nutritional Sciences, Cornell University, Ithaca, NY, USA; ${ }^{4}$ Department of Epidemiology \& Prevention, Wake Forest School of Medicine, Winston-Salem, NC, USA; ${ }^{5}$ Department of Preventive Medicine, Rush University, Chicago, IL, USA; ${ }^{6}$ Department of Internal Medicine, Rush University, Chicago, LL, USA; ${ }^{7}$ Division of Preventive Medicine, University of Alabama at Birmingham, Birmingham, AL, USA; ${ }^{8}$ Department of Preventive Medicine, University of Tennessee Health Science Center, Memphis, TN, USA; 'Department of Internal Medicine, University of lowa Hospitals and Clinics, lowa City, IA, USA.
\end{abstract}

BACKGROUND: Data are limited regarding the influence of diet drink consumption on cardiovascular disease (CVD) outcomes.

OBJECTIVE: We aimed to evaluate the relationship between diet drink intake and cardiovascular events.

DESIGN: We conducted a retrospective cohort study, utilizing data from the national, multicenter Women's Health Initiative Observational Study (WHI OS), recruiting subjects from 1993 to 1998.

PATIENTS: Post-menopausal women with available diet drink intake data, without pre-existing CVD and who survived $\geq 60$ days were included in the study.

MAIN MEAURES: A composite of incident coronary heart disease, heart failure, myocardial infarction, coronary revascularization procedure, ischemic stroke, peripheral arterial disease and CVD death was used as the primary outcome. CVD death and all-cause mortality were secondary outcomes. Adjusted Cox proportional hazards models were used to compare primary and secondary outcomes across diet drink intake strata.

KEY RESULTS: In all, 59,614 women, mean age 62.8 years, were included for analysis. In unadjusted analysis over a follow-up of $8.7 \pm 2.7$ years, the primary outcome occurred in $8.5 \%$ of the women consuming $\geq 2$ diet drinks/day, compared to $6.9 \%, 6.8 \%$ and $7.2 \%$ in the 5-7/week, 1-4/week and 0-3/month groups, respectively. After controlling for other CVD risk factors, women who consumed $\geq 2$ drinks/day had a higher adjusted risk of CVD events (HR 1.3, 95 \% CI 1.1-1.5), CVD mortality (HR 1.5, $95 \%$ CI 1.03-2.3) and overall mortality (HR 1.3, $95 \%$ CI 1.04-1.5) compared to the reference group (0-3 drinks/month).

CONCLUSIONS: This analysis demonstrates an association between high diet drink intake and CVD outcomes and mortality in post-menopausal women in the WHI OS.

Electronic supplementary material The online version of this article (doi:10.1007/s11606-014-3098-0) contains supplementary material, which is available to authorized users.

Received April 21, 2014

Revised August 11, 2014

Accepted October 27, 2014

Published online December 17, 2014
KEY WORDS: diet; cardiovascular diseases; lifestyle; diet drinks; artificial sweeteners.

J Gen Intern Med 30(4):462-8

DOI: $10.1007 / \mathrm{s} 11606-014-3098-0$

(C) Society of General Internal Medicine 2014

\section{INTRODUCTION}

Observational studies have demonstrated an association between sugar-sweetened beverages and obesity, metabolic syndrome, and cardiovascular disease (CVD) outcomes; this association is attributed to increased energy and sugar load. ${ }^{1-5}$ Diet drinks are often consumed as a low-calorie alternative to sugar-sweetened drinks, and one out of every five Americans drinks diet soda every day. ${ }^{6}$ Several population-based studies have demonstrated a positive association between diet drinks and the metabolic syndrome, which in turn is associated with increased risk for CVD. ${ }^{2,7-9}$ However, data are limited as to whether there is an increased CVD risk associated with diet drink consumption. Given the large population directly affected by such an association, this study was performed to evaluate the effect of diet drink consumption on CVD outcomes in the Women's Health Initiative Observational Study (WHI OS) cohort.

\section{METHODS}

The WHI is a multicenter national study that involved 40 centers across 24 states and the District of Columbia and included 161,808 postmenopausal women. ${ }^{10,11}$ It consisted of three overlapping Clinical Trials (CT) including 68,133 women, as well as an Observational Study (OS) enrolling 93,676 subjects. Recruitment to the WHI took place from 1993 to 1998, and enrolled postmenopausal women between 50 and 79 years of age. ${ }^{11}$ The OS cohort was derived from the women who were screened for the clinical trials but were ineligible or unwilling to be randomized, as well as from those 
who responded to a direct invitation to be screened for the OS. ${ }^{12}$ Exclusion criteria (for both the CT and the OS) were the presence of any medical condition with a predicted survival of $<3$ years, concerns about adherence, and active participation in other randomized trials. An effort was made to enroll women of racial and ethnic minority groups, with a target of $20 \%$ of overall enrollment. ${ }^{13}$ No significant sampling issues were noted with regards to participant enrollment. All participants provided written informed consent, and the study protocol was approved by institutional review boards of all participating centers.

The OS cohort was used for analysis for this study. After baseline evaluation at a clinic visit, updates regarding medical histories and selected exposure data were obtained annually by mailed questionnaire. All participants were also invited to a clinic follow-up visit at 3 years after enrollment. ${ }^{13}$ Dietary information was collected using food frequency questionnaires obtained at baseline and follow-up clinic visits. ${ }^{14}$ The questionnaire at follow-up year 3 also included information regarding diet drink consumption over the previous 3 months. Diet drink intake was categorized into nine groups on the questionnaire, ranging from none or less than one per month to more than six drinks per day, with each drink defined as the equivalent of a 12-oz can of beverage. Follow-up ended in 2005 , and ranged from six to ten years, depending on year of enrollment.

\section{Inclusion and Exclusion Criteria}

All women in the OS for whom diet drink intake data was available were included for analysis in this study. Exclusion criteria were (1) presence of any of the following pre-existing diagnoses: coronary heart disease (CHD), heart failure (HF), myocardial infarction (MI), coronary revascularization procedure, ischemic stroke, peripheral arterial disease (PAD) as well as other related cardiovascular and thromboembolic events (angina, carotid artery disease, hemorrhagic stroke, transient ischemic attack, pulmonary embolism and deep vein thrombosis); or (2) a survival of less than 60 days after collection of diet drink consumption data.

\section{Outcomes}

A composite of incident $\mathrm{CHD}, \mathrm{HF}, \mathrm{MI}$, coronary revascularization procedure, ischemic stroke, PAD and CVD death was used as the primary outcome, with time to first occurrence of any of these events being the relevant endpoint. Secondary outcomes were rates of CVD death, as well as death from any cause. Outcomes in the OS were identified through self-report at annual contacts, and specific details were obtained as needed using standardized questionnaires and request of medical records. ${ }^{15}$ Data linkage with the National Death Index was performed to assure completeness of survival data, and causes of death were actively investigated. Adjudication of outcomes was performed by physicians at the Clinical Centers, the Clinical Coordinating Center, and the National Institutes of
Health, using a staged approach. All primary and safety endpoints, as well as a sample of locally adjudicated secondary endpoints, were reviewed centrally. The adjudicating physicians were blinded to any participant information that could potentially result in bias.

\section{Statistical Analysis}

Risk factors and demographic characteristics included baseline self-reported age, race, education, income, body mass index (BMI), smoking, alcohol intake, hormone therapy (HT) use, physical activity, energy intake, salt intake, history of diabetes, ${ }^{16}$ hypertension, or high cholesterol, as well as sugar-sweetened beverage intake. Age was assessed in years as both continuous and categorical (49-59, 60-64, 65-69, $\geq$ 70 ), physical activity was measured as total energy expended, and energy intake was measured as calibrated total calories. ${ }^{17}$

Unadjusted relationships between continuous baseline variables were assessed using t-tests comparing least square means from general linear models, and between categorical variables using the Pearson chi-square test. Occurrence of a CVD event, CVD death and all-cause mortality were measured in years from the date of Visit 3 until the first CVD event, death or until the participant was last known to be alive. Unadjusted differences in incidence of primary and secondary outcomes across diet drink consumption strata were assessed using the Pearson chi-square test. Cox proportional hazards models were used to assess the relationship of diet soda intake with occurrence of the first CVD event or time to death, adjusting for CVD risk factors. ${ }^{18}$ Hazard ratios (HR) and $95 \%$ confidence intervals $(\mathrm{CI})$ were calculated to measure the magnitude of the associations. Four different models were constructed. Model I was unadjusted; Model II was adjusted for age, race, education and income; Model III was adjusted for the variables in Model II plus smoking status, BMI, and a history of diabetes, hypertension and hyperlipidemia; and Model IV was adjusted for the variables in Model III plus alcohol intake, log calibrated energy intake, physical activity, sugar-sweetened beverage intake, salt intake, and HT. Subjects with missing variables were excluded from analysis. All variables were assessed for the proportional hazards assumption before inclusion in the model. Adjusted cardiac event models were stratified on hypertension and high cholesterol, and adjusted mortality models were stratified on hypertension and the history of diabetes, because these variables did not meet the proportional hazards assumption.

Additional sensitivity and exploratory analyses were also performed. In order to decrease the risk of reverse causality, women with a history of diabetes, hypertension, and high cholesterol were excluded and the adjusted risk (Model IV above) for the primary outcome was calculated in the residual healthier population. The impact of missing salt intake and calibrated energy intake data was evaluated by constructing fully adjusted models for the primary outcome after excluding these variables. The effect of BMI on the relationship between relationship of diet drink intake and outcomes was further investigated by using interaction terms for BMI and diet drink 
categories in the fully adjusted models. The role of diet quality was assessed by including Healthy Eating Index-2005 (HEI2005) scores in the model (with salt and energy intake excluded to prevent double counting). HEI-2005 has previously been used in the WHI and other population based cohorts, and has been demonstrated to be associated with both cardiometabolic risk factors and cardiovascular outcomes and mortality. ${ }^{19-23}$ Finally, as the definition of HF in the WHI was sub-optimal, a revised primary outcome that excluded HF was constructed, and adjusted hazard ratios were calculated for the revised primary outcome as well as HF alone.

This manuscript was prepared in accordance with the Strengthening the Reporting of Observational Studies in Epidemiology (STROBE) Statement. ${ }^{24}$ All statistical significance was based on two-tailed tests and $p$ values $\leq 0.050$. Statistical analyses were performed using SAS 9.3 [SAS System for Windows, version 9.3. Cary, NC: SAS Institute. 2002-2010].

\section{RESULTS}

Of 93,676 women who were part of the WHI OS, 59,614 met criteria for this study and were included for analysis (Fig. 1). Among non-deceased women who met other inclusion criteria, 9,946 were excluded due to lack of diet drink data secondary to either absent or partial year 3 follow-up. Those who were excluded had a higher proportion of women older than 70 years of age as well as women of African American or Hispanic race, and had lower income and educational status, compared to women who were included in the analysis.

Almost two-thirds of those included (38,337 women, $64.3 \%)$ fell into the lowest consumption category ( $0-3$ drinks per month), and there were 11,590 (19.4\%), 6,702 (11.2\%) and 2,985 (5.0\%) women who consumed 1-4 drinks per week, 5-7 drinks per week, and two or more drinks per day, respectively (Table 1). Categorization of diet drink intake was constructed in accordance with previous studies and in order to satisfy proportional hazard assumptions. ${ }^{1,2,25}$ The mean follow up was $8.7(\mathrm{SD} \pm 2.7)$ years. The average age of the included cohort was $62.8(\mathrm{SD} \pm 7.2)$ years, and a majority of women $(85.7 \%)$ were white.

Baseline variables differed significantly between the four groups (Table 1). Women who consumed $\geq 2$ diet drinks/day were younger than the other groups, with a mean age of 59.5 years, compared to 63.5 years in the $0-3$ drinks per month group. There was increased prevalence of diabetes and hypertension in the women who consumed the most diet drinks, and they had a higher BMI, a greater proportion of smokers and higher calibrated energy intake. When non-diet drink consumption was assessed across the different diet drink intake strata, no significant difference was found.

\section{Outcomes}

The composite primary outcome occurred in $8.5 \%$ of the women who consumed $\geq 2$ diet drinks/day, compared to 6.9 ,

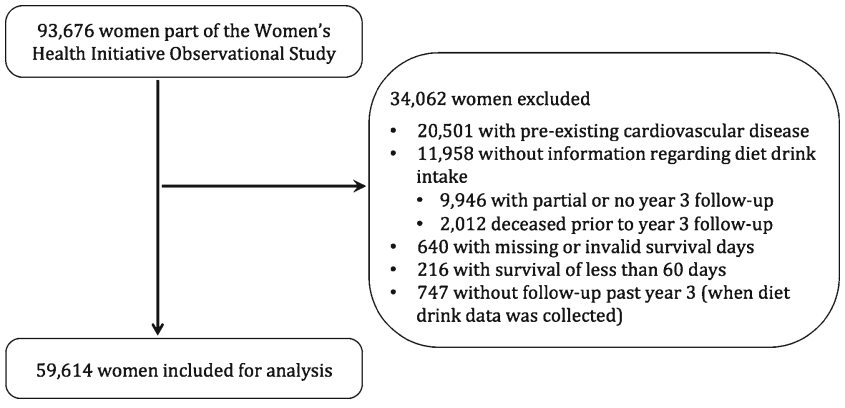

Figure 1 Selection of study cohort.

6.8 and $7.2 \%$ of the women in the 5-7 drinks/week, 1-4 drinks/week and 0-3 drinks/month groups, respectively (Table 2). Unadjusted incidence of the secondary outcomes did not differ markedly between the $\geq 2$ diet drinks/day and $0-3$ diet drinks/month groups, with incidence of CVD death and overall mortality being 1.6 and $7.8 \%$, respectively, in the highest consumption group, and 1.7 and $7.7 \%$ in the lowest (Table 2).

Cox proportional hazard models demonstrated that women who consumed $\geq 2$ drinks/day had a higher unadjusted risk of experiencing CVD events (in the form of the combined endpoint) as compared to those who consumed $0-3$ drinks/month (the reference group), with the difference being statistically significant (Model I; N: 59,614; HR 1.2, 95 \% CI 1.04-1.3) (Table 3). This increased risk among the highest consumption category persisted when the models were adjusted progressively for baseline demographic variables (Model II; N: 55,073; HR 1.6, 95 \% CI 1.4-1.9), common CVD risk factors (Model III; N: 53,037; HR 1.3. $95 \%$ CI 1.2-1.5), and other possible confounders (Model IV; N: 33,619; HR 1.3, $95 \%$ CI 1.1-1.5). No significant difference in risk was found between the other consumption groups and the reference group, with regards to the primary endpoint. There was progressive decrease in sample size with the Cox proportional hazard models secondary to missing data, with Model IV especially limited by missing values for energy and salt intake.

Unadjusted analysis (Model I) did not demonstrate a difference between the $\geq 2$ drinks/day group and the $0-3$ drinks/ month group with regards to either CVD death (N: 59,447; HR $0.9,95 \%$ CI $0.7-1.2$ ) or overall mortality (N: 59,447; HR 1.0, $95 \%$ CI 0.9-1.1) (Table 3). However, with adjustment for baseline demographic characteristics, CVD risk factors and other confounders, there emerged a significant risk of both CVD death (Model II: N 55,073, HR 1.8, 95 \% CI 1.3-2.4; Model III: N 53,037, HR 1.4, 95 \% CI 1.04-1.9; Model IV: N 33,619 , HR 1.5, $95 \%$ CI 1.03-2.3) and overall mortality (Model II: N 55,073, HR 1.5, 95 \% CI 1.3-1.7; Model III: N 53,037, HR 1.4, 95 \% CI 1.2-1.6; Model IV: N 33,619, HR $1.3,95 \%$ CI 1.04-1.5) with consumption of $\geq 2$ diet drinks/ day. Hazard ratios for adjustment covariates for both primary and secondary outcomes are listed in the online appendix. 
Table 1. Baseline Characteristics of Women Included in Analysis

\begin{tabular}{|c|c|c|c|c|c|}
\hline \multirow[t]{2}{*}{ Characteristics } & \multicolumn{5}{|c|}{ Diet drink consumption } \\
\hline & 0-3/month & 1-4/week & 5-7/week & $\geq 2 /$ day & p value* \\
\hline $\mathrm{N}(\%)$ & $38,337(64.3)$ & $11,590(19.4)$ & $6,702(11.2)$ & $2,985(5.0)$ & \\
\hline Age $($ Mean \pm SD) & $63.5 \pm 7.2$ & $62.3 \pm 6.9$ & $61.2 \pm 6.9$ & $59.5 \pm 6.5$ & $<0.0001$ \\
\hline \multicolumn{5}{|l|}{ Race $(\%)$} & $<0.0001$ \\
\hline American Indian & $128(0.3)$ & $28(0.2)$ & $30(0.4)$ & $13(0.4)$ & \\
\hline Asian/Pacific Islander & $1,401(3.7)$ & $270(2.3)$ & $130(1.9)$ & $36(1.2)$ & \\
\hline African American & $2,559(6.7)$ & $720(6.2)$ & $387(5.8)$ & $207(7.0)$ & \\
\hline Hispanic & $1,282(3.4)$ & $363(3.1)$ & $207(3.1)$ & $98(3.3)$ & \\
\hline White & $32,418(84.8)$ & $10,068(87.1)$ & $5880(88.0)$ & $2601(87.4)$ & \\
\hline Other & $441(1.2)$ & $109(0.9)$ & $49(0.7)$ & $22(0.7)$ & \\
\hline \multicolumn{5}{|l|}{ Education $(\%)$} & $<0.0001$ \\
\hline$<$ High school, High school & $10,498(27.6)$ & $3,426(29.8)$ & $1,936(29.1)$ & $893(30.2)$ & \\
\hline Some college & $9,905(26.0)$ & $3,009(26.2)$ & $1,808(27.2)$ & $838(28.3)$ & \\
\hline College graduate, $>$ College & $17,656(46.4)$ & $5,069(44.1)$ & $2,899(43.6)$ & $1,228(41.5)$ & \\
\hline \multicolumn{5}{|l|}{ Income in dollars $(\%)$} & $<0.0001$ \\
\hline$<35,000$ & $12,759(35.8)$ & $3,631(33.5)$ & $1,902(30.2)$ & $925(32.9)$ & \\
\hline $35,000-<75,000$ & $14,852(41.7)$ & $4,643(42.9)$ & $2,772(44.0)$ & $1,193(42.4)$ & \\
\hline$\geq 75,000$ & $8,009(22.5)$ & $2,551(23.6)$ & $1,628(25.8)$ & $693(24.7)$ & \\
\hline \multicolumn{5}{|l|}{$\mathrm{BM} \overline{\bar{M}}(\%)$} & $<0.0001$ \\
\hline$<25 \mathrm{~kg} / \mathrm{m}^{2}$ & $17,692(46.5)$ & $3,882(33.7)$ & $1,986(29.9)$ & $686(23.2)$ & \\
\hline $25-30 \mathrm{~kg} / \mathrm{m}^{2}$ & $12,765(33.6)$ & $4,327(37.6)$ & $2,424(36.5)$ & $1,028(34.7)$ & \\
\hline$>30 \mathrm{~kg} / \mathrm{m}^{2}$ & $7,572(19.9)$ & $3,301(28.7)$ & $2,234(33.6)$ & $1,245(42.1)$ & \\
\hline Smokers $(\%)$ & $1716(4.5)$ & $394(3.4)$ & $303(4.5)$ & $227(7.7)$ & $<0.0001$ \\
\hline \multicolumn{5}{|l|}{ Alcohol intake (\%) } & 0.086 \\
\hline$<0.5$ drinks/week & $22,086(57.7)$ & $6,812(58.9)$ & $3,875(57.9)$ & $1,769(59.3)$ & \\
\hline $0.5-2$ drinks/week & $5,821(15.2)$ & $1,684(14.6)$ & $1,001(15.0)$ & $467(15.7)$ & \\
\hline$>2$ drinks/week & $10,352(27.1)$ & $3,077(26.6)$ & $1,812(27.1)$ & $745(25.0)$ & \\
\hline \multicolumn{5}{|l|}{ HT users $(\%)$} & $<0.0001$ \\
\hline Never & $12,548(32.8)$ & $3369(29.1)$ & $1896(28.3)$ & $878(29.6)$ & \\
\hline Estrogen only & $11,014(28.8)$ & $3623(31.3)$ & $2072(31.0)$ & $943(31.7)$ & \\
\hline Either one or both & $14,565(38.1)$ & $4541(39.3)$ & $2697(40.3)$ & $1136(38.2)$ & \\
\hline Unknown & $129(0.3)$ & $33(0.3)$ & $29(0.4)$ & $14(0.5)$ & \\
\hline Physical activity (total energy expended, MET-hours/week, Mean \pm SD) & $12.0 \pm 13.4$ & $12.1 \pm 13.4$ & $11.6 \pm 13.0$ & $11.9 \pm 13.2$ & 0.38 \\
\hline Calibrated energy intake (kcal, Mean \pm SD) & $2033 \pm 187$ & $2092 \pm 196$ & $2130 \pm 209$ & $2190 \pm 232$ & $<0.0001$ \\
\hline Salt intake (mg, Mean \pm SD) & $2393 \pm 1077$ & $2519 \pm 1098$ & $2601 \pm 1193$ & $2732 \pm 1388$ & $<0.0001$ \\
\hline History of diabetes $(\%)$ & $2636(6.9)$ & $1178(10.2)$ & $810(12.1)$ & $502(16.8)$ & $<0.0001$ \\
\hline History of hypertension (\%) & $9962(26.2)$ & $3281(28.5)$ & $1956(29.4)$ & $927(31.2)$ & $<0.0001$ \\
\hline History of high cholesterol (\%) & $4264(11.4)$ & $1512(13.3)$ & $844(12.8)$ & $384(13.1)$ & $<0.0001$ \\
\hline \multicolumn{5}{|l|}{ Sugar-sweetened beverage intake } & 0.44 \\
\hline None or $<1 /$ month & $23,794(62.1)$ & $7,111(61.4)$ & $4,133(61.7)$ & $1,831(61.3)$ & \\
\hline$<$ Once a day & $12,798(33.4)$ & $3,944(34.0)$ & $2,253(33.6)$ & $1,012(33.9)$ & \\
\hline Daily & $1,745(4.6)$ & $535(4.6)$ & $316(4.7)$ & $142(4.8)$ & \\
\hline
\end{tabular}

SD standard deviation, BMI body mass index, HT hormone therapy

*Unadjusted p value

\section{Sensitivity and Exploratory Analyses}

A history of diabetes, hypertension and high cholesterol was absent in 38,658 women, and these were classified as being healthier at baseline. The hazard ratios for the primary outcome for these were similar to those for the primary analysis; however, the $95 \%$ confidence intervals were wider and did not reach statistical significance (Table 4). Salt and calibrated energy intake data were missing in 18,097 (30.4\%) women, and this was uniformly distributed across the diet drink consumption groups. Excluding salt and calibrated energy intake from the model did not result in a significant change in the

Table 2. Incidence of Primary and Secondary Outcomes Across Diet Drink Consumption Strata

\begin{tabular}{|c|c|c|c|c|c|}
\hline \multirow[t]{2}{*}{ Outcomes } & \multicolumn{5}{|c|}{ Diet drink consumption } \\
\hline & 0-3/month & 1-4/week & 5-7/week & $\geq 2 /$ day & p value* \\
\hline Total subjects & 38,337 & 11,590 & 6,702 & 2,985 & \\
\hline $\begin{array}{l}\text { Cardiovascular events (\%) } \\
\text { Combined }\end{array}$ & $2745(72)$ & $785(68)$ & & & \\
\hline CHD & $1,030(2.7)$ & $\begin{array}{l}785(6.8) \\
279(2.4)\end{array}$ & $\begin{array}{l}462(6.9) \\
159(24)\end{array}$ & $\begin{array}{l}254(8.5) \\
94(3.1)\end{array}$ & 0.010 \\
\hline $\mathrm{HF}$ & $349(0.9)$ & $95(0.8)$ & $53(0.8)$ & $\begin{array}{l}94(3.1) \\
37(1.2)\end{array}$ & $\begin{array}{l}0.053 \\
0.13\end{array}$ \\
\hline MI & $819(2.1)$ & $219(1.9)$ & $122(1.8)$ & $74(2.5)$ & 0.065 \\
\hline Coronary revascularization & $1,039(2.7)$ & $325(2.8)$ & $204(3.0)$ & $111(3.7)$ & 0.008 \\
\hline Stroke & $652(1.7)$ & $192(1.7)$ & $94(1.4)$ & 58 (1.9) & 0.212 \\
\hline PAD & $160(0.4)$ & $49(0.4)$ & $27(0.4)$ & $22(0.7)$ & 0.079 \\
\hline Cardiac death & $642(1.7)$ & $163(1.4)$ & $90(1.3)$ & 47 (1.6) & 0.076 \\
\hline Mortality $(\%)$ & & & & & \\
\hline All cause death & $2,970(7.7)$ & $789(6.8)$ & $445(6.6)$ & $233(7.8)$ & 0.003 \\
\hline
\end{tabular}

CHD coronary heart disease, HF heart failure, MI myocardial infarction, PAD peripheral arterial disease

*Unadjusted p values ( $\chi^{2}$ test) 
Table 3. Hazard Ratios for Primary and Secondary Outcomes Across Diet Drink Consumption Strata

\begin{tabular}{|c|c|c|c|}
\hline \multirow[t]{3}{*}{ Models } & \multicolumn{3}{|l|}{ Outcomes } \\
\hline & \multirow{2}{*}{$\frac{\text { Cardiovascular events }}{\text { HR }(95 \% \text { CI })}$} & \multirow{2}{*}{$\frac{\text { Cardiac death }}{\text { HR }(95 \% \mathrm{CI})}$} & \multirow{2}{*}{$\frac{\text { Overall death }}{\text { HR }(95 \% \text { CI })}$} \\
\hline & & & \\
\hline Model I* & $\mathrm{N}: 59,614$ & $\mathrm{~N}: 59,447$ & $\mathrm{~N}: 59,447$ \\
\hline $0-3 /$ month & & & \\
\hline 1-4/week & $0.9(0.9-1.02)$ & $0.8(0.7-0.99)$ & $0.9(0.8-0.9)$ \\
\hline $5-7 /$ week & $1.0(0.9-1.1)$ & $0.8(0.6-0.99)$ & $0.8(0.8-0.9)$ \\
\hline$\geq 2 /$ day & $1.2(1.04-1.3)$ & $0.9(0.7-1.2)$ & $1.0(0.9-1.1)$ \\
\hline Model II ${ }^{\dagger}$ & $\mathrm{N}: 55,073$ & N: 55,073 & $\mathrm{~N}: 55,073$ \\
\hline $0-3 /$ month & 1.0 & 1.0 & 1.0 \\
\hline $1-4 /$ week & $1.0(0.96-1.1)$ & $1.0(0.8-1.2)$ & $1.0(0.9-1.1)$ \\
\hline $5-7 /$ week & $1.1(1.0-1.3)$ & $1.1(0.9-1.4)$ & $1.1(1.0-1.2)$ \\
\hline$\geq 2 /$ day $_{*}$ & $1.6(1.4-1.9)$ & $1.8(1.3-2.4)$ & $1.5(1.3-1.7)$ \\
\hline Model III" ${ }^{\star}$ & $\mathrm{N}: 53,037$ & N: 53,037 & N: 53,037 \\
\hline $0-3 /$ month & 1.0 & 1.0 & 1.0 \\
\hline $1-4 /$ week & $1.0(0.9-1.1)$ & $0.9(0.8-1.1)$ & $1.0(0.9-1.1)$ \\
\hline 5-7/week & $1.0(0.9-1.1)$ & $1.0(0.8-1.3)$ & $1.1(1.0-1.2)$ \\
\hline$\geq 2 /$ day & $1.3(1.2-1.5)$ & $1.4(1.04-1.9)$ & $1.4(1.2-1.6)$ \\
\hline Model IV $\mathrm{IV}^{\S}$ & $\mathrm{N}: 33,619^{\|}$ & $\mathrm{N}: 33,619^{\|}$ & $\mathrm{N}: 33,619^{\|}$ \\
\hline $0-3 /$ month & 1.0 & 1.0 & 1.0 \\
\hline 1-4/week & $1.0(0.9-1.1)$ & $0.9(0.7-1.2)$ & $1.0(0.9-1.1)$ \\
\hline 5-7/week & $1.1(0.9-1.2)$ & $0.9(0.7-1.3)$ & $1.1(0.9-1.2)$ \\
\hline$\geq 2 /$ day & $1.3(1.1-1.5)$ & $1.5(1.03-2.3)$ & $1.3(1.04-1.5)$ \\
\hline
\end{tabular}

HR hazard ratios, CI confidence intervals

*Model I was unadjusted

${ }^{*}$ Model II was adjusted for age, race, education and income

Model III was adjusted for the variables in Model II plus smoking status, BMI, and a history of diabetes, hypertension and hyperlipidemia

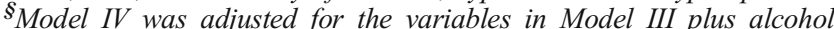
intake, log calibrated energy intake, physical activity, sugar-sweetened beverage intake, salt intake, and hormone therapy. Adjusted cardiac event models were stratified on hypertension, and high cholesterol and adjusted mortality models were stratified on hypertension and the history of diabetes because these variables did not meet the proportional hazards assumption

"The majority of loss of patients in model 4 was secondary to missing data for salt intake and calibrated energy

hazard ratios for the primary outcome (HR for $\geq 2$ diet drink/ day group: 1.3 ; $95 \%$ CI 1.1-1.5). Addition of the HEI-2005 score in the model also did not lead to any notable change in the association between diet drink consumption and CVD events (HR for $\geq 2$ diet drinks/day group: 1.3; $95 \%$ CI 1.1-1.5).

A significant unadjusted and adjusted interaction was observed between baseline BMI and diet drink consumption for the primary outcome ( $p=0.003$ for both) (Online appendix). On adjusted analysis, consumption of $\geq 2$ diet drinks/day continued to be significantly associated with occurrence of CVD events in women with BMI $\geq 30$ (HR 1.3, $95 \%$ CI 1.11.6) and BMI $<25$ (HR 1.7, $95 \%$ CI 1.3-2.4), while the association was not statistically significant in women with BMI 25-30 (HR 1.2, 95 \% CI 0.9-1.5).

Finally, exclusion of HF from the primary outcome did not change the hazard ratios significantly compared to the primary analysis, and these continued to be statistically significant for the $\geq 2$ diet drinks/day group (Online appendix).

\section{DISCUSSION}

This study demonstrates an association between regular daily intake of two or more diet drinks and CVD outcomes and
Table 4. Adjusted Hazard Ratios for Primary Outcome Across Diet Drink Consumption Strata for Primary Analysis, for Baseline

Healthy Women, with Salt Intake and Calibrated Energy Intake Excluded, and with Healthy Eating Index-2005 Included

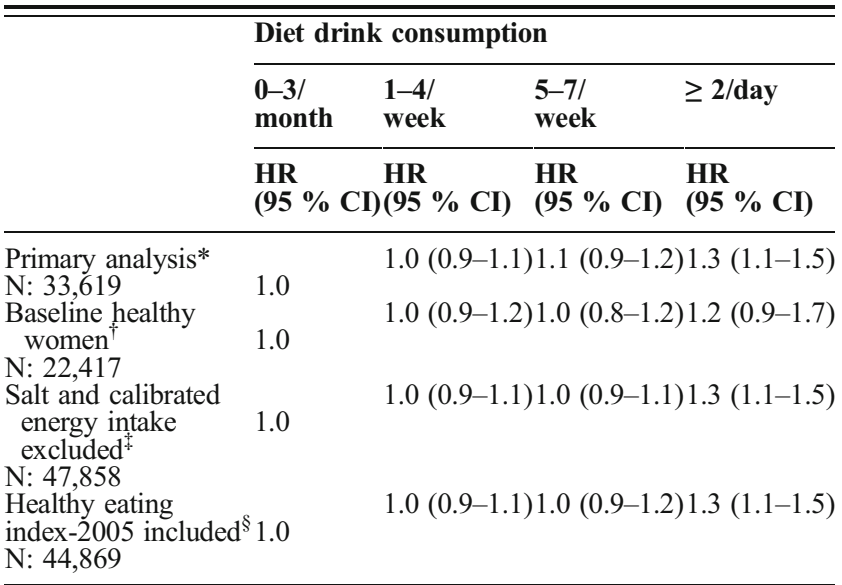

HR hazard ratio, CI confidence interval

*Model IV: Adjusted for age, race, education, income, smoking status, BMI, diabetes, hypertension, hyperlipidemia, alcohol intake, log calibrated energy intake, physical activity, sugar-sweetened beverage intake, salt intake, and hormone therapy

${ }^{4}$ Model IV; Included women without a history of diabetes, hypertension, and high cholesterol

*Adjusted for Model IV excluding salt intake and calibrated energy intake

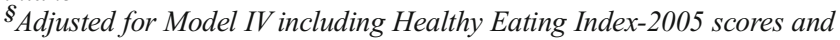
excluding salt intake and calibrated energy intake

mortality in post-menopausal women. In our analysis, women who consumed $\geq 2$ diet drinks/day had a $30 \%$ higher adjusted risk of CVD events as well as overall mortality compared to those with an intake of $0-3$ diet drinks/month.

Our study, with nearly 60,000 subjects, is one of the largest studies evaluating diet drink consumption and outcomes. Our findings are concordant with the results of the only previous report that expressly evaluated the risk of CVD events with diet drink intake. ${ }^{25}$ Gardener et al. used the Northern Manhattan Study (NOMAS) cohort, and included 2,564 subjects (64\% female). They found daily diet drink consumption to be associated with an increased adjusted risk of CVD events (incident stroke, MI or vascular death). The overall event rate was about $23 \%$ over a mean follow-up of 9.8 years, which is significantly higher than the total event rate of $7.1 \%$ over 8.7 years in our study; some of this may be explained by the presence of both men and women in the NOMAS subject population, as well as more ethnic diversity compared to the WHI cohort.

The results are also supported by other observational data that have shown a link between diet drink consumption and metabolic syndrome. Three separate reports, involving patients from the Framingham Heart Study, the Atherosclerosis Risk in Communities study and the Multi-Ethnic Study of Atherosclerosis demonstrated significantly increased rates of incident metabolic syndrome among subjects who were frequent consumers of diet drinks. ${ }^{2,8,9}$ Another report that analyzed data from the San Antonio Heart Study noted a greater incidence of obesity and a significantly higher 
increase in BMI with increasing intake of artificially sweetened beverages, with an apparent dose response relationship between the amount of artificially sweetened beverages consumed and weight gain. ${ }^{7}$ As both metabolic syndrome and obesity are important risk factors for CVD, this may contribute in part to the higher incidence of CVD events in this population.

Exact pathophysiologic mechanisms that would explain the association of weight gain, development of metabolic syndrome, and increased CVD events with diet soda consumption are still unclear. One hypothesis is that artificial sweeteners may increase the desire for sugar-sweetened, energy-dense beverages/foods. Experimental data from animal (rat) models suggests that consumption of products containing artificial sweeteners may disrupt the correlation between sweet taste and the energy content of foods (thus interfering with fundamental homeostatic and physiological processes). ${ }^{26}$ Another study investigating the functional magnetic resonance imaging response to sucrose (a nutritive sweetener) and saccharin (a nonnutritive sweetener) in diet soda drinkers versus non-diet soda drinkers found alterations in the reward processing of sweet taste in individuals who regularly consume diet soda. ${ }^{27}$

An alternative explanation for this association could be confounding by dietary patterns or incomplete adjustment for confounders. In a recent study, diet beverage consumers defined as having a healthy diet had a lower risk of metabolic syndrome. ${ }^{28}$ Analysis from the Health Professionals FollowUp Study presented evidence of an association between consumption of sugar-sweetened beverages and increased CHD risk and intermediate biomarkers, but no associations were found for artificially sweetened beverage intake. ${ }^{29}$ However, the study noted that artificially sweetened beverage consumption was associated with healthy lifestyle traits and higher overall diet quality, which may suggest a role of dietary pattern in the determination of outcomes. In addition, individuals attempting to restrict energy intake and control weight may be more likely to consider artificially sweetened beverages, a factor that may influence CVD risk factor associations. ${ }^{30}$ The sensitivity analysis evaluating women without diabetes, hypertension and high cholesterol at baseline was done to address some of these questions. While the confidence intervals widened (with loss of statistical significance), the hazard ratios did not change significantly. Additionally, inclusion of the HEI-2005 score, which is a validated tool for quantifying dietary quality, also did not result in a significant change in the association between diet drink consumption and CVD events. Finally, the interaction between BMI and diet drink intake noted in our analysis is an interesting finding that bears further investigation, especially given other recent data that highlight the role of body weight in this setting. ${ }^{31}$

Limitations of this study include its observational nature, and the fact that it involved retrospective analysis of data not collected expressly for the purpose of this paper. It also involves a specific population, that of post-menopausal women, and thus may not be generalizable to other populations. As the differences in demographic characteristics between women excluded for missing diet drink data and the included women demonstrate, presence of selection bias cannot be ruled out. Due to limited data collection regarding diet drink intake after the baseline evaluation, the analysis does not take into account changes in consumption pattern over the course of follow-up. Finally, the association seen in this study does not translate into causality, and may be due to confounding variables that were not examined in multivariate analysis, or may not even be clearly defined as being confounders in this relationship as of yet.

In conclusion, this study suggests an association between consumption of two or more diet drinks per day and adverse CVD events, as well as increased mortality. However, further evaluation with other clinical studies, animal models and even molecular and pharmacologic analyses is needed to confirm or disprove this link, and to assess a possible causal relationship between diet drink intake and increased CVD risk.

Funding Sources: The Division of Cardiovascular Medicine at the University of Iowa Hospitals \& Clinics and the Women's Health Initiative provided partial funding for this manuscript.

Conflict of Interest: The authors declare that they do not have a conflict of interest.

\section{Relationship with Industry: None.}

Corresponding Author: Ankur Vyas, MD; Division of Cardiovascular MedicineUniversity of Iowa Hospitals Clinics, 200 Hawkins Dr., Int. Med. E316-1 GH, Iowa City, IA 52242, USA (e-mail: ankurvyas7@gmail.com).

\section{REFERENCES}

1. Fung TT, Malik V, Rexrode KM, Manson JE, Willett WC, Hu FB. Sweetened beverage consumption and risk of coronary heart disease in women. Am J Clin Nutr. 2009;89(4):1037-1042. doi:10.3945/ajcn.2008.27140.

2. Dhingra R, Sullivan $\mathbf{L}$, Jacques PF, et al. Soft drink consumption and risk of developing cardiometabolic risk factors and the metabolic syndrome in middle-aged adults in the community. Circulation. 2007;116(5):480488. doi:10.1161/CIRCULATIONAHA.107.689935.

3. Malik VS, Popkin BM, Bray GA, Després J-P, Hu FB. Sugar-sweetened beverages, obesity, type 2 diabetes mellitus, and cardiovascular disease risk. Circulation. 2010;121(11):1356-1364. doi:10.1161/ CIRCULATIONAHA. 109.876185.

4. Qi Q, Chu AY, Kang JH, et al. Sugar-sweetened beverages and genetic risk of obesity. N Engl J Med. 2012;367(15):1387-1396. doi:10.1056/ NEJMoa1203039.

5. Schulze MB, Manson JE, Ludwig DS, et al. Sugar-sweetened beverages, weight gain, and incidence of type 2 diabetes in young and middle-aged women. JAMA. 2004;292(8):927-934. doi:10.1001/jama.292.8.927.

6. Fakhouri THI, Kit BK, Ogden CL. Consumption of diet drinks in the United States, 2009-2010. NCHS Data Brief. 2012;109:1-8.

7. Fowler SP, Williams K, Resendez RG, Hunt KJ, Hazuda HP, Stern MP. Fueling the obesity epidemic? Artificially sweetened beverage use and long-term weight gain. Obesity. 2008;16(8):1894-1900. doi:10.1038/oby.2008.284.

8. Nettleton JA, Lutsey PL, Wang Y, Lima JA, Michos ED, Jacobs DR. Diet soda intake and risk of incident metabolic syndrome and type 2 diabetes in the Multi-Ethnic Study of Atherosclerosis (MESA). Diabetes Care. 2009;32(4):688-694. doi:10.2337/dc08-1799.

9. Lutsey PL, Steffen LM, Stevens J. Dietary intake and the development of the metabolic syndrome: the atherosclerosis risk in communities study. Circulation. 2008;117(6):754-761. doi:10.1161/CIRCULATIONAHA.107.716159. 
10. Rossouw JE, Hurd S. The Women's Health Initiative: recruitment complete-looking back and looking forward. J Womens Health. 1999;8(1):3-5.

11. Hays J, Hunt JR, Hubbell FA, et al. The Women's Health Initiative recruitment methods and results. Ann Epidemiol. 2003;13(9 Suppl):S1877. doi:10.1016/S1047-2797(03)00042-5

12. Langer RD, White E, Lewis CE, Kotchen JM, Hendrix SL, Trevisan M. The Women's Health Initiative Observational Study: baseline characteristics of participants and reliability of baseline measures. Ann Epidemiol. 2003;13(9 Suppl):S107-21. doi:10.1016/S10472797(03)00047-4.

13. The Women's Health Initiative Study Group. Design of the Women's Health Initiative clinical trial and observational study. Control Clin Trials. 1998;19(1):61-109.

14. Patterson RE, Kristal AR, Tinker LF, Carter RA, Bolton MP, AgursCollins T. Measurement characteristics of the Women's Health Initiative food frequency questionnaire. Ann Epidemiol. 1999;9(3):178-187.

15. Curb JD, Mctiernan A, Heckbert SR, et al. Outcomes ascertainment and adjudication methods in the Women's Health Initiative. Ann Epidemiol. 2003;13(9 Suppl):S122-8. doi:10.1016/S1047-2797(03)00048-6.

16. Margolis KL, Lihong $\mathbf{Q}$, Brzyski R, et al. Validity of diabetes self-reports in the Women's Health Initiative: comparison with medication inventories and fasting glucose measurements. Clin Trials. 2008;5(3):240-247. doi: $10.1177 / 1740774508091749$.

17. Neuhouser ML, Tinker L, Shaw PA, et al. Use of recovery biomarkers to calibrate nutrient consumption self-reports in the Women's Health Initiative. Am J Epidemiol. 2008;167(10):1247-1259. doi:10.1093/ aje/kwn026.

18. Cox DR. Regression models and life-tables. J R Stat Soc Ser B. 1972;34:187-220.

19. Nicklas TA, O'Neil CE, Fulgoni VL. Diet quality is inversely related to cardiovascular risk factors in adults. J Nutr. 2012;142(12):2112-2118. doi:10.3945/jn.112.164889.

20. Chiuve SE, Fung TT, Rimm EB, et al. Alternative dietary indices both strongly predict risk of chronic disease. J Nutr. 2012;142(6):1009-1018. doi:10.3945/jn.111.157222.

21. George SM, Ballard-Barbash R, Shikany JM, et al. Better postdiagnosis diet quality is associated with reduced risk of death among postmenopausal women with invasive breast cancer in the Women's Health Initiative.
Cancer Epidemiol Biomark Prev. 2014;23(4):575-583. doi:10.1158/10559965.EPI-13-1162.

22. Guenther PM, Reedy J, Krebs-Smith SM. Development of the healthy eating index-2005. J Am Diet Assoc. 2008;108(11):1896-1901. doi:10.1016/j.jada.2008.08.016.

23. Guenther PM, Reedy J, Krebs-Smith SM, Reeve BB. Evaluation of the healthy eating index-2005. J Am Diet Assoc. 2008;108(11):1854-1864. doi:10.1016/j.jada.2008.08.011.

24. von Elm E, Altman DG, Egger M, Pocock SJ, Gøtzsche PC, Vandenbroucke JP. The Strengthening the Reporting of Observational Studies in Epidemiology (STROBE) statement: guidelines for reporting observational studies. Ann Intern Med. 2007;147(8):573-577. doi:10.7326/0003-4819-147-8-200710160-00010.

25. Gardener H, Rundek T, Markert M, Wright CB, Elkind MSV, Sacco RL. Diet soft drink consumption is associated with an increased risk of vascular events in the Northern Manhattan Study. J Gen Intern Med. 2012;27(9):1120-1126. doi:10.1007/s11606-011-1968-2.

26. Swithers SE, Davidson TL. A role for sweet taste: calorie predictive relations in energy regulation by rats. Behav Neurosci. 2008;122(1):161173. doi:10.1037/0735-7044.122.1.161.

27. Green E, Murphy C. Altered processing of sweet taste in the brain of diet soda drinkers. Physiol Behav. 2012;107(4):560-567. doi:10.1016/ j.physbeh.2012.05.006.

28. Duffey KJ, Steffen LM, Van Horn L, Jacobs DR, Popkin BM. Dietary patterns matter: diet beverages and cardiometabolic risks in the longitudinal Coronary Artery Risk Development in Young Adults (CARDIA) Study. Am J Clin Nutr. 2012;95(4):909-915. doi:10.3945/ ajcn.111.026682.

29. de Koning L, Malik VS, Kellogg MD, Rimm EB, Willett WC, Hu FB. Sweetened beverage consumption, incident coronary heart disease, and biomarkers of risk in men. Circulation. 2012;125(14):1735-41-S1. doi:10.1161/CIRCULATIONAHA.111.067017.

30. Elfhag K, Tynelius P, Rasmussen F. Sugar-sweetened and artificially sweetened soft drinks in association to restrained, external and emotional eating. Physiol Behav. 2007;91(2-3):191-195. doi:10.1016/ j.physbeh.2007.02.005.

31. Bleich SN, Wolfson JA, Vine S, Wang YC. Diet-beverage consumption and caloric intake among US adults, overall and by body weight. Am J Public Health. 2014:e1-e7. doi:10.2105/AJPH.2013.301556. 